\section{Serum uric acid and leg ulcers*}

\author{
Rodrigo Araldi Nery ${ }^{1}$ \\ Thelma Larocca Skare ${ }^{2,3}$
}

DOI: http:/ / dx.doi.org/10.1590/abd1806-4841.20198140

Dear Editor,

Chronic leg ulcers (LU) are those that do not heal in four to six weeks, remaining stagnant in the inflammatory phase. ${ }^{1}$ The influx of polymorphonuclear cells to the wound site, with release of lysosomal enzymes and oxygen free radicals, contributes to cell injury and disease maintenance, impairing healing. ${ }^{2}$

Uric acid (UA) has historically been associated with situations of increased oxidative stress, playing an important role in maintaining the inflammatory process on surfaces where there is tissue necrosis, and in triggering the inflammatory cascade. ${ }^{2}$

Fernandez et al. ${ }^{3}$ demonstrated that UA levels in the wound fluid of patients with LU are high and that its concentration is proportional to ulcer severity. The same authors also observed that xanthine oxidase, an enzyme that catalyzes the production of UA, is active in chronic wound secretions. ${ }^{2}$

After approval of the local Committee of Ethics in Research and signed consent, we studied 70 patients (mean age $63.3 \pm 12.1$ years, $60 \%$ women) to observe whether serum UA levels influence tissue healing and the presence of pain in venous LU.

Data collection was done in two visits, three months apart. During this time, all patients received standard treatment. During both visits, we recorded the ulcers' appearance, number and size. We also recorded the degree of pain using a visual analogue scale (VAS) ranging from 0 to 10 , where $0=$ no pain and $10=$ worst pos-

Received 31 January 2018

Accepted 27 June 2018.

Work conducted at the Hospital Universitário Evangélico de Curitiba, Faculdade Evangélica do Paraná, Curitiba (PR), Brazil. Financial support: None.

Conflict of interest: None.

1 Department of Vascular Surgery, Hospital Universitário Evangélico de Curitiba, Faculdade Evangélica do Paraná, Curitiba (PR), Brazil.

2 Discipline of Rheumatology, Medicine Course, Faculdade Evangélica do Paraná, Curitiba (PR), Brazil.

3 Rheumatology Service, Hospital Universitário Evangélico de Curitiba, Faculdade Evangélica do Paraná, Curitiba (PR), Brazil.

MAILING AdDRESS:

Thelma Larocca Skare

E-mail: tskare@onda.com.br

C2019 by Anais Brasileiros de Dermatologia sible pain. The ulcerated area was calculated by multiplying the measurements, in centimeters, of the two main axes of the wound. For patients with multiple wounds, the final area was calculated by summing the individual measurements. Presence of granulation tissue, fibrin, hyperkeratosis and necrosis was noted. UA was measured using the dry chemistry method, during the first visit, after 8 hours of fasting. Values up to $6.2 \mathrm{mg} / \mathrm{dL}$ for women and up to 8.5 $\mathrm{mg} / \mathrm{dL}$ for men were considered normal.

The degree of healing was evaluated by comparing the number and area of the ulcers between the first and second visits.

In this sample, $25.7 \%$ of the subjects were hyperuricemic. At the first visit, the median number of ulcers/patient was 1.0; the median area of ulcer/patient was $18 \mathrm{~cm}^{2}$, and the mean VAS pain was 3.0. Granulation tissue was present in $98.5 \%$, fibrin in $42.8 \%$, hyperkeratosis in $2.8 \%$, and none had necrotic tissue. At this first visit, a correlation was found between the degree of pain and the level of $\mathrm{UA}(\mathrm{p}=0.02$, Rho $=0.27,95 \% \mathrm{CI}=0.03-0.47)$, but not between pain and the area or number of ulcers $(\mathrm{p}=\mathrm{ns})$. Figure 1 shows an association study of serum UA with ulcer characteristics.

There was no correlation between serum UA and the differences (delta) in the number and size of ulcers (all $p=n s)$. The difference in pain showed a correlation with UA ( $p=0.01$; Rho $=0.29$; $95 \% \mathrm{CI}=0.05-0.50)$.

This study shows that uricemia is associated with pain and increased fibrin formation in leg ulcers, but it does not affect the ulcer's healing rate.

Pain is a common complaint in patients with LU, and it is one of the main contributors to loss of life quality in this context. ${ }^{1}$ In gout, pain is caused by the release of mediators that stimulate nociceptors during crystal phagocytosis. ${ }^{4}$ However, it has been shown that urate crystals alone, without leukocyte infiltration, can also cause pain. ${ }^{4}$ In animals, UA promotes dose-dependent pain that can be prevented by treatment with vanilloid receptor-1 blockers, demonstrating a neurogenic component in the genesis of this symptom. ${ }^{4}$

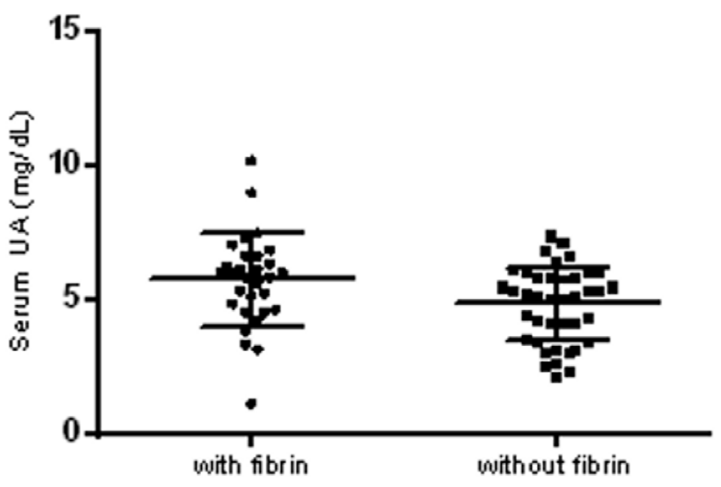

Figure 1: Association study of serum uric acid (UA) with fibrin presence in leg ulcers (with fibrin, mean UA of $5.7 \pm 1.7 \mathrm{mg} / \mathrm{dL}$; without fibrin, mean UA of $4.8 \pm 1.3 \mathrm{mg} / \mathrm{dL} ; \mathrm{p}=0.01$ ). No association was found with hyperkeratosis $(p=0.42)$. Presence of granulation and necrosis could not be associated with UA levels due to the small size of one of the samples 
We have no explanations for the association of serum UA levels with pain in LU; however, it is possible to hypothesize that an individual with higher levels of UA in the blood may also have higher UA levels in the ulcerated wound.

The association of UA with presence of fibrin was also observed. The accumulation of fibrin in tissues results from fibrinogen escape through capillary pores enlarged by increased local venous pressure. Burnand et al. ${ }^{5}$ suggested that pericapillary fibrin affects the diffusion of oxygen and nutrients, favoring ischemia and impairing healing. Thus, it is possible to theorize that elevated levels of UA are associated not only with the presence of fibrin but also with oxygenation impairment and diminished healing. However, we have not been able to demonstrate this last hypothesis.

We conclude that UA levels are associated with pain severity and with the local formation of fibrin in LU, demonstrating that this radical is somehow involved in the pathophysiology of chronic ulcers. $\square$

\section{REFERENCES}

1. Parker CN, Finlayson KJ, Shuter P, Edwards HE. Risk factors for delayed healing in venous leg ulcers: a review of the literature. Int J Clin Pract. 2015;69:967-77.

2. Fernandez ML, Upton Z, Shooter GK. Uric acid and xanthine oxidoreductase in wound healing. Curr Rheumatol Rep. 2014;16:396.

3. Fernandez ML, Upton Z, Edwards H, Finlayson K, Shooter GK. Elevated uric acid correlates with wound severity. Int Wound J. 2012;9:139-49.

4. Hoffmeister C, Trevisan G, Rossato MF, de Oliveira SM, Gomez MV, Ferreira J. Role of TRPV1 in nociception and edema induced by monosodium urate crystals in rats. Pain. 2011;152:1777-88.

5. Burnand KG, Whimster I, Naidoo A, Browse, NL. Pericapillary fibrin in the ulcerbearing skin of the leg: the cause of lipodermatosclerosis and venous ulceration. Br Med J (Clin Res Ed). 1982;285:1071-2.

\section{AUTHORS' CONTRIBUTIONS \\ Rodrigo Araldi Nery \\ (iD) ORCID 0000-0001-8805-983X \\ Approval of the final version of the manuscript, Elaboration and writing of the manu- script, Obtaining, analyzing and interpreting the data, Critical review of the literature, Critical review of the manuscript \\ Thelma Larocca Skare \\ 0000-0002-7699-3542 \\ Statistical analysis, Approval of the final version of the manuscript, Conception and planning of the study, Elaboration and writing of the manuscript, Effective participation in research orientation, Critical review of the literature, Critical review of the manuscript}

How to cite this article: Nery RA, Skare TL. Serum uric acid and leg ulcers. An Bras Dermatol. 2019;94(3):369-70.

\section{CASE LETTERS}

Wells' syndrome: the importance of differential diagnosis*

\author{
Lilian Geronimo Brasileiro ${ }^{1}$ \\ Marilda Aparecida Milanez Morgado de Abreu ${ }^{1}$ \\ Renato Soriani Paschoal ${ }^{2}$
}

DOI: http:/ /dx.doi.org/10.1590/abd1806-4841.20197840

Dear Editor,

We describe three female patients with recurrent episodes of skin lesions that were diagnosed with Wells' syndrome (WS). The first case we report is a 37-year-old female patient that has presented with red, infiltrated, circular plaques with high and precise borders for the past two years. Some lesions show a central dissected blister, as well as erythematous maculas on the periphery; lesions are asymptomatic and located on the face and upper limbs (Figure 1). The episodes resolved spontaneously in approximately eight weeks and reoccurred, on average, every two months. The second case is a 34-year-old white woman who has shown recurrent erythematous-edematous plaques and papules with central desquamation for the last five years; the lesions first appeared after her first pregnancy. Initially, the lesions were limited to the left forearm, however they later affected the sternal region, being more intense in each crisis. The third case is a 42 -year-old white female patient that has presented pruritic erythematous-edematous plaques with papules for 6 months in the cervical region and asymptomatic erythematous maculas on the left shoulder. (Figure 2). Peripheral eosinophilia was only observed in one patient. The anatomopathological findings of the three patients showed eosinophilic inflammatory infiltrate compatible with WS (Figure 3). In all three cases, treatment with oral steroids led to the disappearance of the lesions but relapses occurred after the drug was discontinued. Late diagnosis of WS is common,

Received 07 November 2017

Accepted 07 August 2018.

* Work conducted at the Hospital Regional de Presidente Prudente, Universidade do Oeste Paulista, Presidente Prudente (SP), Brazil Financial support: None. Conflict of interest: None.

1 Dermatology Service, Hospital Regional de Presidente Prudente, Universidade do Oeste Paulista, Presidente Prudente (SP), Brazil. 2 RSP Dermatologia, Ribeirão Preto (SP), Brazil.

MAILING AdDRESS:

Lilian Geronimo Brasileiro

E-mail: dermatolilianbrasileiro@gmail.com

C2019 by Anais Brasileiros de Dermatologia 